\title{
A lifestyle monitoring system to support (in)formal caregivers of people with dementia: Analysis of users need, benefits, and concerns
}

\author{
Erik Zwierenberg BSc Eng ${ }^{\mathrm{a}, *}$ \\ Henk Herman Nap PhD ${ }^{b}$ \\ Dirk Lukkien MSc ${ }^{b}$ \\ Lotte Cornelisse $\mathrm{MSc}^{\mathrm{b}}$ \\ Evelyn Finnema $\mathrm{PhD}^{\mathrm{c}}$ \\ Ate Dijkstra Med $\mathrm{PhD}^{\mathrm{d}}$ \\ Mariët Hagedoorn $\mathrm{PhD}^{\mathrm{a}}$ \\ Robbert Sanderman $\mathrm{PhD}^{\mathrm{e}}$
}

\begin{abstract}
${ }^{a}$ Department of Health Psychology, University Medical Center Groningen, University of Groningen, Groningen, the Netherlands; 'bilans, Dutch Centre of Expertise for Long-term Care,

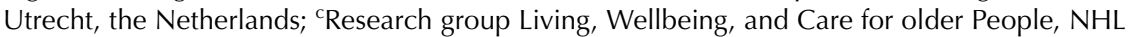
Stenden University of Applied Sciences, Leeuwarden, the Netherlands; ${ }^{\mathrm{d}}$ Research group iHuman, NHL Stenden University of Applied Sciences, Leeuwarden, the Netherlands; ${ }^{\text {Depart- }}$ ment of Health Psychology, University Medical Center Groningen, University of Groningen, Groningen, the Netherlands. Department of Psychology, Health and Technology, University of Twente, Enschede, the Netherlands; *Corresponding author: e.zwierenberg@umcg.nl
\end{abstract}

E. Zwierenberg, H.H. Nap, D. Lukkien, L. Cornelisse, E. Finnema, A. Dijkstra, M. Hagedoorn, R. Sanderman. A lifestyle monitoring system to support (in)formal caregivers of people with dementia: Analysis of users need, benefits, and concerns. Gerontechonology 2018;17(4):194-205; https://doi.org/10.4017/gt.2018.17.4.001.00 Introduction Dementia is a syndrome that predominantly affects people in old age. Many older people with mild to moderate dementia live at home alone. When dementia poses problems, they must rely on informal caregivers, who have lives of their own in other places, as well as on professional home care organized by case managers. Assistive technologies, such as lifestyle monitoring, are being developed to assist informal caregivers and case managers by making remote caregiving possible. In a study conducted in the northern Netherlands, people with dementia were provided with a lifestyle monitoring system consisting of activity sensors in the home connected to an online platform. This study is intended to generate insight into needs, benefits, and concerns relating to a lifestyle-monitoring system to help informal caregivers and case managers provide care to people with dementia who are living alone. The key research questions are as follows: Which needs, benefits, and concerns are reported by informal caregivers and case managers who use lifestyle monitoring? What does the collected information indicate about the adoption of lifestyle monitoring? Methodology In the study, 63 lifestyle-monitoring systems were installed in 63 homes of people with mild to moderate dementia who were living alone. We guaranteed that the system could be used without charge until the end of the trial. We conducted telephone interviews with informal caregivers (50) near the date on which the system was installed, and again when it had been in use for approximately 300 days (41). We also interviewed case managers (13) at the beginning and at the end of the project (7). Each interview lasted about 30 minutes and consisted of closed and open questions about topics including expectations, experiences, quality of life, and care. The study took place in the rural area of the northern Netherlands from February 2015 to June 2016. Four homecare organizations provided lists of participants to include in the study. Results/conclusion The results indicate that informal caregivers perceive lifestyle monitoring as a support tool that fills a need in the provision of care for people with dementia and that its benefits outweigh the 
concerns. Lifestyle monitoring makes it possible to expand the informal care network such that care duties can be shared among more people, thereby relieving informal caregivers of a sense of constant responsibility.

\section{Keywords: older adults, home care, assistive/home monitoring technology, eHealth, telehealth, dementia}

\section{INTRODUCTION}

As the population in developed countries ages, the number of people experiencing age-related health problems in the final stages of life is increasing. In the province of Friesland (located in the northern Netherlands), the number of people with dementia (PWD) is expected to undergo an increase of $71 \%$ by 2030 (from 10,500 in 2012 to 18,000 in 2030). Given that the overall population in Friesland (approx. 650,000) is not expected to change in the coming decades, the decline in the size of the working population will make it difficult to fill the need for nursing staff (Chorus, Perenboom, Hoffstetter, \& Stadlander, 2014).

Friesland recognizes the worldwide policy guidelines on 'aging in place' and delaying admission to intramural long-term care (ILTC) as long as possible (Moïse, Schwarzinger, \& Um, 2004). This also corresponds to the fact that most older people have a strong desire to continue their normal lives in their own familiar environments, and that they apply strategies to adapt to changes associated with normal aging (Atchley, 1989; Wahl, Iwarsson, \& Oswald, 2012). When syndromes like dementia occur, however, informal or formal care becomes necessary.

Informal caregivers of PWD often experience considerable pressure to help the people for whom they are providing care achieve the goal of staying at home. Information about the changing patterns of daily life of PWD, which might indicate health problems, could be valuable to informal caregivers and lessen their burden. Assistive technologies or telecare and, more specifically, lifestyle monitoring (Gibson et al., 2016) are intended to record daily activity patterns of PWD living at home alone.

Dementia manifests itself in various symptoms that signal a neurological disorder: aphasia (loss of language), apraxia (loss of motoric skills), and agnosia (loss of the ability to recognize objects). People with dementia also experience increasing difficulty in abstract thinking and complex behavior. In addition, they may also develop a tendency to wander from home, and their sleeping patterns are often disturbed (e.g. with later bedtimes) (Novitzky et al., 2015). An increase in the frequency of restroom visits is another sign of health problems (Eriksson, Gustafson, Fagerstrom, \& Olofsson, 2010).
Lifestyle-monitoring systems use sensors to record the movement of PWD in their homes. The sensors can help caregivers to monitor behavior that could signal problems in everyday functioning (as described above). In most cases, sensors are placed in each room of the house, as well as at the front and back doors. Information from the sensors is collected centrally, and can be presented to informal and formal caregivers through a cloud-based platform to a web browser on personal computers, tablets or smartphones. Lifestyle monitoring is a nonintrusive system that requires no action from the PWD. Caregivers can receive information about the whereabouts of PWD without being in the same location with them. The information that is collected can help informal caregivers to make decisions about the care provided. Several small-scale studies have been conducted on lifestyle-monitoring systems. Although the results are largely encouraging, none of the systems has been widely adopted, and additional research is necessary (Chi \& Demiris, 2015).

The complexity and intensity of care for PWD increases with the severity of the health problems, as does the amount of time spent on informal and formal care. In addition to adding to the body of knowledge concerning the progress of dementia, the information provided by lifestyle-monitoring systems help to assess whether it is possible and safe for individual PWD to continue living at home alone. The use of such systems could provide informal caregivers with a greater sense of control, thereby reducing their level of stress.

In this study, the users of the lifestyle-monitoring system were informal caregivers and case managers. Case managers are nurses who support PWD from the time of diagnosis until the end of their lives. Their activities include organizing and providing advice about the care to be given. To generate insights into the real-world experiences of people using a lifestyle-monitoring system, we installed such systems in the homes of PWD and interviewed their informal caregivers and case mangers about their experiences with the assistive technology. To analyze their feedback, we labelled the results using a semi-open coding technique. The labels and topics were compared to the acceptance factors (Yusif, Soar, \& HafeezBaig, 2016) and barriers to the adoption of assistive technologies (Yusif, Soar, \& Hafeez-Baig, 2016), thus generating qualitative insight into 
the needs, benefits, and concerns of system users. These insights might increase the likelihood of potential users to adopt lifestyle monitoring. The key research questions are as follows: Which needs, benefits, and concerns are reported by informal caregivers and case managers who use lifestyle-monitoring systems? What does the information collected indicate about the adoption of lifestyle monitoring?

\section{Methodology \\ Participants and procedure}

We included informal caregivers who were providing care for people with very mild cognitive decline (Stage 2 ) to moderately severe cognitive decline (Stage 5), according to the Global Deterioration Scale (Reisberg, Ferris, de Leon, \& Crook, 1982). A second inclusion criterion for informal caregivers was that the PWD for whom they were providing care were living at home alone. The case managers of the participating informal caregivers were also included in the study.

In the province of Friesland, all PWD are registered and assigned to a case manager at the time of diagnosis. In all, 25 case managers are responsible for 1350 PWD. Because the lifestyle-monitoring system is intended for use by people who are living at home alone, only one fourth of the informal caregivers of PWD (about 340) could be considered potential users of the system.

To find participants, we provided training to all of the case managers with regard to the possibilities offered by the lifestyle-monitoring system. They subsequently explained these opportunities to the informal caregivers of selected PWD during regular visits. After providing this information, the case managers asked the informal caregivers whether they (along with the PWD for whom they were providing care) would be willing to participate in the study. The budget allowed for the inclusion of up to 100 systems. In all, 63 informal caregivers agreed to participate. Due to the timeframe of the project, we stopped including participants after March 2016.

We chose to interview only informal caregivers and their case managers because they are the users of the lifestyle monitoring system. Due to cognitive decline, people with dementia experience many problems using technology (Hedma, Lindqvist, \& Nygard, 2016). Lifestyle-monitoring systems were therefore developed in such a way

Table 1. Participants per user group by measurement over time

\begin{tabular}{lcc}
\hline Number of interviewees (n) & \multicolumn{2}{c}{ Measurement in time } \\
\cline { 2 - 3 } & T0 & T1 (after 300 days) \\
\hline Informal caregivers (n) & 50 & 41 \\
\hline Case managers (n) & 13 & 7 \\
\hline
\end{tabular}

that PWD do not need to operate them. The first individual interviews with most of the participating informal caregivers (50) and case managers (13) were conducted by telephone, and a few participants were interviewed in the homes of the PWD for whom they were providing care. Of the 63 informal caregivers who agreed to participate, only 50 could be interviewed. The reasons for the 13 people who could not be interviewed varied (e.g. the PWD had already been moved to ILTC, or the informal caregivers could not be reached, had changed their minds and did not wish to be interviewed, or had withdrawn their intention to use the system). Only 13 out of the 25 participating case managers were interviewed, because these 13 case managers served the 50 informal caregivers and PWD included in the study. The next step consisted of installing the lifestyle-monitoring systems.

We interviewed the informal caregivers (41) and case managers (7) again 300 days after the first interview. Nine informal caregivers could not be interviewed the second time, mostly due to changes in the care provided. Due to organizational problems, five case managers could not be interviewed the second time (Table 1).

The informal caregivers $\left(n=50, M_{\text {age }}=54.9\right.$, SDage $=7.5,35$ female) were interviewed twice. The time $\left(\mathrm{M}_{\text {months }}=6.5, \mathrm{SD}_{\text {months }}=1.5\right)$ between the two interviews was long enough to allow the respondents to internalize the operations and use the system routinely. There was considerable variations in the distance between the homes of the PWD and informal caregivers $\left(\mathrm{M}_{\mathrm{km}}=37.4, \mathrm{SD}_{\mathrm{km}}=\right.$ 55.6). Informal caregivers travelled by car $(n=29)$, bicycle $(n=13)$, or other means of transportation, and many $(\mathrm{n}=39)$ had professional obligations in addition to their care duties. Most of the informal caregivers $(n=29)$ visited the PWD for whom they were providing care once or twice a week. The informal caregivers were regular users of desktop computers $(n=30)$, laptops $(n=22)$, smartphones $(n=35)$, and tablets $(n=25)$.

\section{Research methodology}

To answer the research questions, we adopted an in-depth qualitative interview method. The study started in July 2015 (T1) and ended in June 2016 (T2). Semi-structured interview guides were developed for the interviews, with an interview design for informal caregivers at T1 and T2, and another interview design for the case managers at T1 and T2. The interview designs consisted of closed and open questions. Questions for informal caregivers concerned the following topics: experience with technology, tasks to perform as a caregiver, the situation of the PWD, lifestyle monitoring, and quality 
of care. Questions for case managers concerned the following topics: experience with technology, lifestyle of clients and informal caregivers, experience and expectations regarding lifestyle monitoring, quality of life, and quality of care. The interviews lasted about 30 minutes each, and were conducted by researchers and students who had been trained for this purpose. Interview guides were used in all interviews. Answers were recorded in writing during the interviews and copied to a spreadsheet program for further analysis.

\section{Data Analysis}

A spreadsheet program was used to conduct the qualitative analysis. The interview data were labelled using a semi-open coding technique. Feedback based on the closed questions was labelled by a researcher using a simple codebook based on the topics of the questions. To minimize single-researcher bias, a second (different) researcher sampled the data and verified whether the results described were proper interpretations of the data. Feedback based on the open questions was added to the codebook and clustered into topics according to a cross-case analysis session involving a group of four researchers, who compared and discussed the feedback. The labels were added when consensus was reached. In this case as well, feedback on the open questions was compared to the additional labels, and to each other (level of cases) by one researcher, with the other researcher sampling and checking the data to increase reliability.

\section{Material}

The Livind lifestyle-monitoring system (www. livind.nl) was used for the purposes of this study and participants got it for free during the trial period. The Dutch government provided a Grant to Vilans and Livind to organize a field trail with care-organizations to gain more experience with the concept of lifestyle monitoring. Livind was one of the two major lifestyle monitoring developers at that moment in the Netherlands. Informal caregivers and case managers (nurses

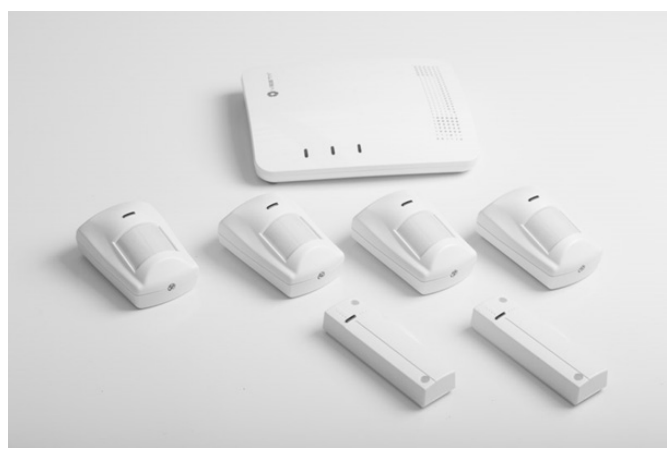

Figure 1. Picture of PIR, door/window sensors and gateway to internet router who serve as the primary contact for PWD) can consult the Livind website or Webapp on a tablet or smartphone at any time to learn the daily pattern and whereabouts of those for whom they are providing care. Passive infrared (PIR) sensors (Figure 1) are placed in every room of the house (5-7 sensors). In addition, door-contact sensors are placed on the front and back doors. Given the differences in the interior design of individual homes, the placement of sensors must be customized. A professional system installer visited each respondent included in the study and discussed the locations for the sensors with the informal caregiver. When an animal (for example a dog) was in the house, placement of the sensors was critical to prevent wrong signals. This was done to ensure that the system would work without problems from the start of the study. We made sure that the lifestyle-monitoring system was a supplement and did not replace any other assistive technologies (e.g. personal alarm systems) already in use. All humans produce body heat, and the PIR sensor in a given room detects this heat. The sensor also measures the intensity of movement of the person in the room. Both signals (location and movement) are collected.

The PIR and door sensors are equipped with batteries and have a wireless connection to a gateway. The gateway is connected to a router (internet) in the house to transmit the information that has been collected to a centralized database. There, the signals are stored and prepared for nursing staff or informal caregivers to access through the internet. After the user has received authorization to consult the data, the information is displayed on demand on a web-based scalable dashboard (website), which can be accessed using a personal computer, tablet, or smartphone. Thresholds (alarms) can be set to trigger a text message or email. A helpdesk was available to provide support in the event of problems with configuration or the installation of the sensors.

\section{Software configuration and system operation}

The information collected through the sensors is stored in a cloud-based database, thus enabling longitudinal data collection. Information on patterns of everyday life (room, time, movement), including day-night rhythm, is stored, and consecutive days can be compared and analyzed. Alarms (triggers) can be set two weeks after the installation of the lifestyle-monitoring system, based on the specific daily patterns of individual PWD. The PIR and door sensors can detect changes in activity patterns that will trigger alarms, and deviations over time provide relevant input for adaptations in the care process for PWD.

The dashboard (Figure 2) displays the measurement of each sensor in a table row. The longitu- 


\section{A Iifestyle monitoring system}

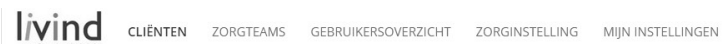

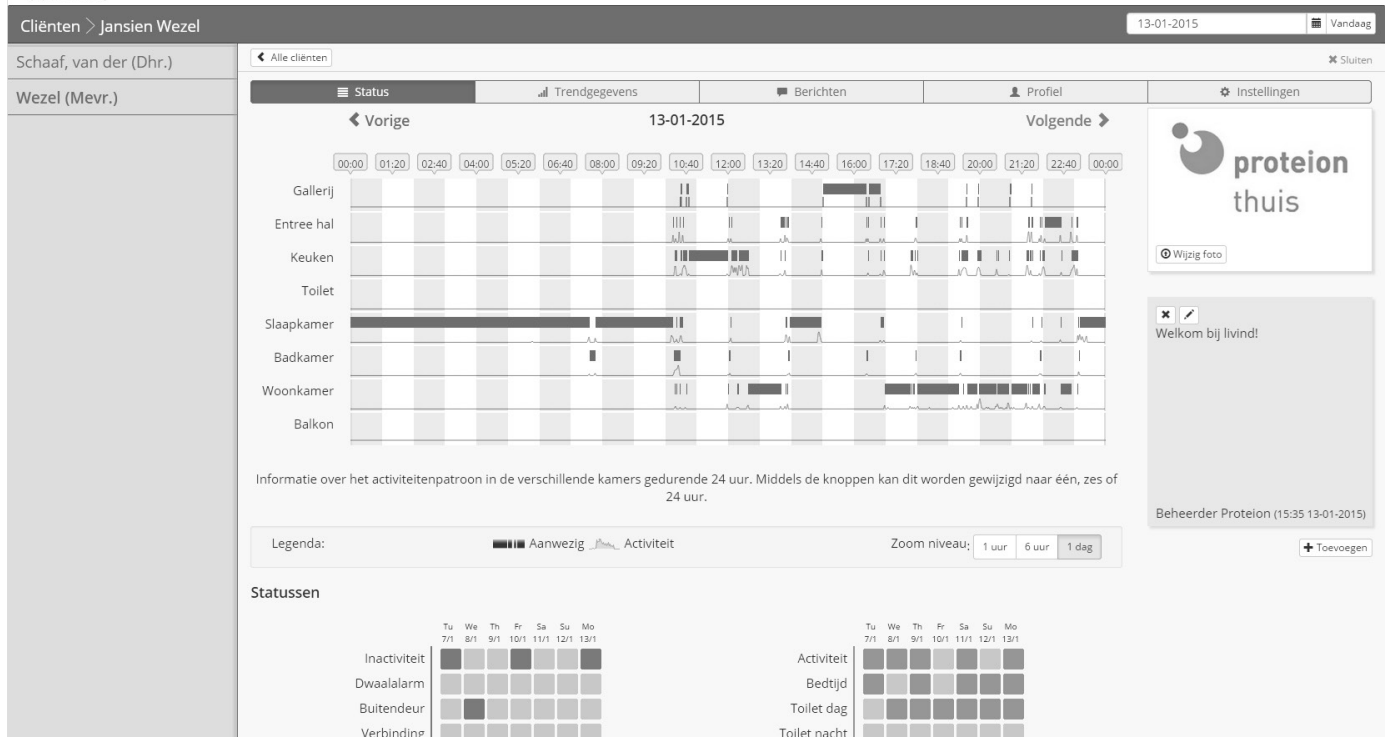

Figure 2. Online dashboard of the lifestyle-monitoring system

dinal (time) data are displayed in columns. The thick line represents the location of the sensor that detects the presence of the PWD.

The most recent seven days of sensor data are displayed at the bottom of the dashboard. The color of the sensor on a certain day depends on the following factors: inactivity, false alarms, outside door, connection, restroom during the day, restroom at night, bathroom and kitchen. Red bars represent critical alarms, with orange representing deviations from the set values, green indicating that everything is normal, and gray indicating the absence of measurements.

The data trend (Figure 3) during a certain period is presented ( 10 days, 1 month, 3 months, and 6 months), including the following information:

(1) Time spent sleeping

(2) Activity (sum score of movements in 24 hours)

(3) Restroom visits (8:00-22:00)

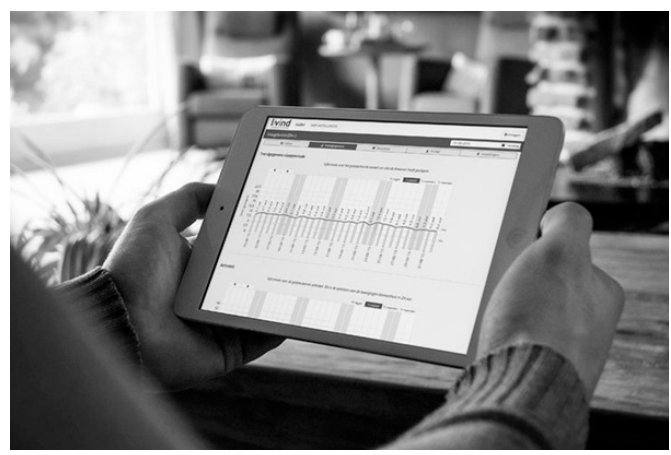

Figure 3. Periodic trend
(4) Restroom visits (22:00-8:00)

(5) Hours per day that the resident has been outside of the house

\section{Privacy, anonymity, and ethics}

Each participating informal caregiver and case manager signed an informed-consent document. The PWD were not interviewed. Any other technologies that were being used for the safety and security of the PWD were left unchanged. The lifestyle-monitoring system was used supplementary to any other technologies in use. The results were anonymized to make it impossible to trace them back to the people who were interviewed. To ensure privacy, we use numbers instead of names to identify the participants: informal caregivers (IC1-IC63) and case managers (CM1CM13).

\section{Results}

After clustering the data to labels and grouping them into topics, the results could be categorized as needs (3.2), benefits (3.3), and concerns (3.4). The three categories used to present the results of our study are based on the pre-implementation acceptance factors, as described by Peek and colleagues (Peek et al., 2014), and the adoption factors described by Yusif and colleagues (Yusif et al., 2016). In Section 3.1, we describe several characteristics of the participating informal caregivers, along with information concerning the use of the lifestyle-monitoring system.

\section{Need for lifestyle monitoring}

The needs expressed by the participants have been grouped into labels, which have been 
Table 2. Need for lifestyle monitoring

\section{Topic \\ (3.2.1) Expectations of the lifestyle-monitoring system}

\section{(3.2.2) Changes in care deployment}

\section{Label}

Knowledge of caregivers about lifestyle monitoring

Better overview of activity when PWD is alone

Need for global insight and early signaling of deterioration

Alert when something happens

Expect to improve anticipation of the care question

Not expected to provide more care, but to provide care at other times

Few actual adjustments to care after six months

Customized care according to caregivers

Customized care according to case managers

\section{(3.3.3) Changed time before admission into intramural long-term care}

Predictably difficult to predict

Many expectations that it will be possible to continue living at home for longer

No effect on continuing to live at home longer

Support for determining whether it would be wise to continue living at home grouped into topics. The results are presented in Table 2. In the following sections, several quotations are presented (in italics) to illustrate the data behind the labels.

\section{Expectations of the lifestyle monitoring system} Informal caregivers often have doubts about the information they receive directly from the PWD for whom they provide care, and they expect to be able to use the information provided by the lifestyle-monitoring system as a cross-check. Our respondents indicated that they expected the system to provide them with objective information concerning the daily activity pattern, as well as an overall overview and signals of changes (e.g. confusion, syndrome-related deviations, midnight wandering and decreasing use of the kitchen). "We had questions about what she was doing. When you're there, nothing's happening, but we'd like to know what they're looking for

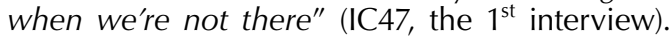
They also expected the system to provide information to support their assessment of the extent to which the PWD are able to live independently at home. "Dementia is a known process. It's irreversible. At some point, he won't be able to live alone, and I hope to be able to notice that in time" (IC11, the $1^{\text {st }}$ interview). Overall, they expect the value of the information provided by the lifestyle-monitoring system to exceed that of the effort needed to operate it.

A lifestyle-monitoring system is not a substitute for an alarm system. The informal caregivers participating in this study differed with regard to their expectations of what would happen if a major health risk were to occur. Some of them expected to receive alarms whenever the PWD wander away. Others had a more realistic view and did not expect that the system would trigger alarms calling for immediate follow-up. They did expect to receive signals for prolonged deviations due to falls or changes in restroom behavior.

In general, the expectations of the case managers with regard to the lifestyle-monitoring system were the same as those of the informal caregivers. The following were among the expectations they expressed: recognition of deviations in patterns, early signaling of deterioration on the part of PWD:"...see deviations in time, adjust care times, and respond to them so that they can live at home longer" (CM8, the $1^{\text {st }}$ interview). One case manager noted that the system configuration is important for filtering problems and preventing the occurrence of false alarms. In addition, case managers indicated expecting that the system would support informal caregivers, but that they would not have the time to follow all of their clients through the system. According to their expectations, if informal caregivers are better informed, they will be able to provide better information to the case managers.

\section{Changes in care deployment}

Informal caregivers reported that they expect the system to signal deterioration in an early phase, thus allowing for adjustments in care. Case managers shared this expectation. Informal caregivers also expected that, although the PWD would require the same amount of care, the system would improve the efficiency and effectiveness of the organization of care. Some case managers agreed and expressed the expectation that the system would reduce the need for professional care.

After six months of use, some informal caregivers noted that there had been no change in the care. "We indicated that she was restless at night, but that was a one-time occurrence, so the care 
was not adjusted" (IC34, the $2^{\text {nd }}$ interview). Other informal caregivers did report perceiving changes in care. Case managers used the information provided by the system to optimize the care. "The best example is Mrs. [...]. Normally, much more care would be devoted to checking on her health. This is not done now, however, because the monitoring system shows that her auto-pilot is strong enough to do things well. She wants as few intruders as possible, and we wish to respect that. The usual recommendation is to increase the amount of care provided, but the lifestyle monitoring makes this unnecessary" $\left(\mathrm{CM} 5\right.$, the $2^{\text {nd }}$ interview). Case managers report that problems like urinary infection, which is common for PWD, was discovered early a few times.

Changes in the time before admission into intramural long-term care (ILTC)

Most informal caregivers indicated that it is difficult to predict whether the system would allow the

PWD for whom they were providing care to continue living at home longer. The information provided by the system could reassure them that it is still possible to live at home, although it could also provide a clearer demonstration that it is no longer

Table 3. Benefits (actual and expected) of lifestyle monitoring

Topic

Label

(3.3.1) Reassuring the A measure of peace caregiver

Understanding what happens when the informal caregiver and the PWD are not together

Understanding of the day-night rhythm

Becoming familiar with the system

Insufficient confidence in or even stress due to lifestyle monitoring

(3.3.2) Changes in the Verify what the PWD says.

role of the informal Responding to the situation

caregiver

Remote involvement

Supportive, albeit low-impact action

Amount of contact

(3.3.3) Sense of safety Not all informal caregivers inform their relatives about the system for of the PWD

(3.3.4) User experiences with the lifestyle-monitoring system possible. "It could go both ways. [...] Another conclusion could be that there are many activities that are in conflict with staying at home longer" (IC53, the $1^{\text {st }}$ interview). One case manager expressed a similar opinion. Most of the informal caregivers expected that the system would support living at home longer. This expectation was shared by case managers. Some of the informal caregivers and case managers were under the impression that the PWD would have already gone into ILTC had it not been for the system. "It will not delay the time at which she will eventually have to live somewhere else, but we now have more and better arguments to support the choice" (IC5, the $2^{\text {nd }}$ interview). Other informal caregivers did not expect the system to have any influence on the timing of moving into intramural long-term care.

\section{Benefits of lifestyle monitoring}

The benefits expressed by the participants have been grouped into labels, which have been grouped into topics (Table 3). The insights generated by the results are described in the following sections, illustrated by examples from the data (indicated in italics).
Reassuring the caregiver

When starting to use the system, some of the in formal caregivers needed to become accustomed to being able to observe every movement of the PWD for whom they were providing care. After they had grown accustomed to this, they report feeling reassured, which they appreciated, k n o w in g what was h a p p e n ing with the PWD. "It gives a 
measure of peace. We can cross-check what our mother tells us, and we can keep an eye on Mom from a distance. That sounds negative, but it's comforting to know that she did not leave the door open at night. And because you stay calm, you do not take measures as soon as possible. She will not be able to stay at home for much longer." (IC5, the $2^{\text {nd }}$ interview). Case managers concurred with regard to the sense of reassurance reported by the informal caregivers, and they noted that the system provided informal caregivers with greater control of the situation. "It provides a little extra control when I'm home and not with my grandmother. Then I can also see if she is still in bed after she has been put to bed, or how often she has gone to the restroom. It is an additional care moment" (IC9, the $1^{\text {st }}$ interview). Some informal caregivers were especially reassured to know how the daynight rhythm was developing. Others reported that the system had caused an increase in stress, due to delayed signaling, inaccuracy, or supposed emergencies. "The family has become restless in response to a number of signals from the system. As a result, they felt obliged to go to her at very inconvenient times. It does not add anything, and the family is likely to drop the lifestyle-monitoring system" (CM7, the $2^{\text {nd }}$ interview).

\section{Changes in the role of the informal caregiver}

Informal caregivers used the system to assess what the PWD were doing, and they often used the information to cross-check the oral information provided by the PWD. They also used the information provided by the system to address particular issues (e.g. frequency of restroom use, not taking a walk during the day, not using the kitchen). This information makes it possible for informal caregivers to respond in a more informed way. "Now you know what she has been doing when you talk with her. Because she's getting worse, she says things that are not correct. With the lifestyle monitor, you know what she really did" $^{\prime \prime}$ (IC41, the $2^{\text {nd }}$ interview).

Informal caregivers who were living at a greater distance from the PWD for whom they were providing care reported that the system had made a positive contribution to their ability to provide informal care. "We - who are living on a distance - are more involved than we would be otherwise and are able to relieve our brothers and sisters - who live nearby and are doing most of the caregiving - a little bit" (IC5, the $2^{\text {nd }}$ interview).

Although not all of the informal caregivers reported changing their ways of working, they did indicate that the system had provided them with support, reassurance, and confirmation. The frequency of the visits that informal caregivers made to the PWD for whom they were providing care changed due to the information provided by the system. "I no longer have to walk past her house late in the evening. Before, I went to her house five times a week, and now twice a week. I can see when there is activity. This reduces the pressure on the informal caregiver. Apart from that, dementia increases, and the demand for care increases along with it" (IC10, $2^{\text {nd }}$ interview).

\section{Sense of safety on the part of PWD}

At the start, some PWD were suspicious of the system, although this decreased over time. Informal caregivers were under the impression that the PWD were pleased that someone was looking after them. Some informal caregivers told the PWD for whom they were providing care that the system was for their own safety. Other informal caregivers did not tell the PWD anything about the system, for fear of negative reactions. Some informal caregivers told the PWD that the system serves a different purpose. Most of the PWD were not aware of the system, even when the caregiver did give an explanation. "At the very least, he knows that he will be able to stay in his apartment longer because we can follow him through the lifestyle-monitoring system. He had been taken to a nursing home earlier, and had not liked it there. He does feel that the implementation of the system has now made it possible for him to stay at home for longer" (IC7, the $2^{\text {nd }}$ interview). Ethical and moral implications of these findings will be addressed in the discussion.

\section{User experiences with the lifestyle-monitoring system}

After using the system for 300 days, informal caregivers reported that they checked the system regularly to monitor and evaluate the daynight rhythm of the PWD, as presented by the lifestyle-monitoring dashboard. They checked the nightly activity of the PWD; the times at which they got out of bed, left the house, and returned home; activity in the house, and visits to the restroom. "Does he stay in bed at night, or does he walk around, and how often? Based on that information, I have contacted the GP and other professionals a number of times" (IC15, the $2^{\text {nd }}$ interview). Some informal caregivers monitored specific activities. One informal caregiver explained that she and her mother had agreed that the balcony was too dangerous. She uses the system to monitor whether her mother is adhering to the agreement.

One of the reasons given by informal caregivers for monitoring the activity data irregularly was irregular working hours. Several informal caregivers indicated that they had used the system actively in the first few weeks in order to check on the daily activity pattern, but that the frequency had decreased over time. Some informal caregivers indicated dissatisfaction with the system and 
the configuration of the alarms. The informal caregivers used the system to watch for trends or to find out more about alarms that they had received from the system. "Such a system lets you keep an eye on the long term in a better way. The separate signals are not very meaningful if you look at them individually. But when you look at the trends, it provides a bit of reassurance. Sometimes, she might sleep a little more than she does other times but, in general, it does not change so much" (IC2, the $2^{\text {nd }}$ interview).

Case managers checked the system once every $6-8$ weeks, just before visiting their clients. They checked deviations from general patterns. Some case managers expressed that they did not wish to be responsible for alarms generated by the system. One case manager reported using the system less frequently. "I take a look at the screen every two months. I also use it to prepare for meetings with home care, to see what is going on, and things like that." (CM5, the $2^{\text {nd }}$ interview). The overall impression is that informal caregivers initially use the lifestyle-monitoring system intensively, eventually adjusting their use of the system to their day-to-day habits, as it satisfies their need to know what is going on, or they stop using the system because the problems that it creates outweigh its benefits.

\section{Concerns relating to lifestyle monitoring}

The concerns expressed by the participants have been grouped into labels, which have been grouped into topics (Table 4). The insights generated by the results are described in the following sections, illustrated by examples from the data (indicated in italics).

Customer-satisfaction points relating to the system Informal caregivers reported a variety of positive aspects of the lifestyle-monitoring system. Examples included convenient emails, graphs, and traffic-light (green, orange, red) reports, which they perceived as insightful and easy to read. In

Table 4. Concerns relating to lifestyle monitoring

\section{Topic}

(3.4.1) Customersystem satisfaction points of the

\section{Label}

Many notifications - often the same information

Adjusting of notifications to the individual lifestyle

The system does not provide timely alarms in case of deviations.

Uncertainty regarding what the system indicates

The system generates false and incorrect notifications when there are multiple people in the house.

Placement of the various sensors

Additional points for improvement

(3.4.2) Privacy is not of paramount importance addition to the positive aspects, they mentioned several problems and ideas for improving the lifestyle-monitoring system. The six most prominent points are listed below.

(1) The system generates too many reports, which often contain the same information. Informal caregivers feel that the information is not reliable.

(2) The configuration of the reports should have more options for customizing them to the situations of the PWD being monitored.

(3) The system does not issue alarms in a timely manner (e.g. when the PWD wanders away from home). Informal caregivers perceive a need for such alarms.

(4) Some informal caregivers are uncertain about what the system indicates. Reports or alarms that cannot be explained fuel distrust in the system.

(5) The system generates false reports and alarms when guests are present in the PWD's home.

(6) The location of the sensors should be more customized than it currently is.

\section{Privacy is not of paramount importance}

Privacy did not seem to be a major issue from the perspective of the informal caregivers, but this does not mean that it is not a major issue. Questions remain concerning whether the installation of monitoring systems is humane and acceptable from a moral and ethical point of view. Most of the informal caregivers were positive about the objectivity of the signals generated by the system, which did not include camera images that would have major implications for the privacy of PWD. Other informal caregivers were of the opinion that the system did constitute at least some violation of privacy. Several stated that, although some infringement of privacy was involved, they did not experience it as problematic. The sense of safety and assurance provided by the system outweighed the privacy issue. "She does not mind. In the case of dementia, it is necessary to make some concessions in terms of privacy in order to ensure security" (IC10, the $1^{\text {st }}$ interview).

\section{Discussion}

The aim of the present study was to test a lifestyle monitoring system for informal caregivers and case managers who support patients with dementia. We examined the lifestyle-monitoring system Livind, which was introduced to caregivers and case

\section{No privacy-related concerns}

Who has access to the dashboard and what are the actions taken with the information? 
managers in order to generate an insight into the various needs, benefits, concerns, and requirements of potential users of lifestyle-monitoring systems. In the perception of the users, lifestyle monitoring fulfils a need and the users appreciated the benefits of the system to such an extent that they even wished to continue using it after the end of the research period, taking in mind recommendations for improvements.

The system was developed in such a way that its operations do not require any action from the PWD. According to the results of our study, most PWD tend to forget about the system within a few days of its installation in their homes. The results of this study indicate that it helps to signal changes in daily activity that many PWD are no longer capable of mentioning. The system signals lifestyle changes to nursing staff and informal caregivers, allowing them to act on any of the changes that might occur. At the start informal caregivers and case managers were very optimistic about the potential of lifestyle monitoring to enable the PWD to live at home longer. At the end of the study, they noted that they were indeed better informed and prepared for the decision to enter intramural longterm care (ILTC) when it would become necessary.

It is important to note that providing informal care for PWD has many positive aspects, including meaningfulness, companionship, and the opportunity to improve someone's quality of life (ADI Consortium, 2009). The informal caregivers participating in our study noted that they had become more connected to the people for whom they were providing care and were able to talk with them about what they had seen on the lifestyle-monitoring system. Most of the end-users we consulted were positive about the "functionality and benefits" of lifestyle monitoring in the care for PWDs. One remark in this regard is that the system appears to provide too much information (alarms) at the wrong time and that the information is not easily accessible ("ease of use"). Satisfied users considered the system "suitable for daily use," although some mentioned that they used it less often than they did in the beginning ("perception of no need"). Most of the PWD involved in this study forgot about the existence of the system within a few days ("no stigma") and "dependence" on the system did not appear to be an issue for the informal caregivers.

The results also indicate that informal caregivers regard lifestyle monitoring as a support tool that can reduce stress and reduce the burden of care for PWD (Reeder et al., 2013). Furthermore, the system could allow the expansion of the informalcare network and provide relatives of PWD with a means with which to delegate care more efficiently and effectively, in addition to relieving the infor- mal caregivers of a constant sense of responsibility.

From the wider literature it is clear that caring for PWD can be a burden that poses personal, emotional, and physical challenges. The burden becomes heavier as the disease progresses. The increasing severity of cognitive disorders and the resulting threats to the independence of the PWD are exacerbated by behavioral symptoms which heighten the distress, anxiety, and depression experienced by informal caregivers and may even jeopardize their physical health (Aguglia et al., 2004; Laparidou, Middlemass, Karran, \& Siriwardena, 2016). Hence, assistive technology may help out. One systematic review (Reeder et al., 2013) concludes that lifestyle-monitoring technology is "promising", but that further research is necessary in order for such technology to be classified as "effective". According to Nijhof (2013), additional research is needed to establish whether it helps to delay the admission of PWD into institutional long-term care (ILTC).

With regard to the "privacy dilemma", our results from informal caregivers indicate that the impact of a lifestyle-monitoring system on privacy is more than compensated for by the perceived sense of safety that the information provides. "Trust" seems to be a problem at first, with PWD being suspicious about the sensors in their homes. Another aspect of trust has to do with the concerns of informal caregivers regarding who will have access to and use the data. Both "trust" and "privacy" are closely aligned with ethical and moral aspects of monitoring people from a distance. In this study, we learned that users (i.e. informal caregivers and case managers) focused more on the utility of the system and did not directly address any ethical and moral considerations.

In conclusion, the results of this project and others described in the literature, are generally positive, but not yet conclusive. A review of the research on the effects of the broad range of assistive technologies with regard to relieving the burdens perceived by informal caregivers, sustaining the cognitive and functional capabilities of PWD, and supporting their ability to continue living at home supports this notion but also issues the need for further research (Chi \& Demiris, 2015; Gibson et al., 2016; N. Nijhof, van GemertPijnen, Woolrych, \& Sixsmith, 2013). Additional research in which also quantitative outcomes (e.g. satisfaction among users, time to admission to specialized care and the like) over time are assessed is needed in order to confirm the positive results on the concept of lifestyle monitoring. Our study generated information about the PWD through their informal caregivers and case managers. In future research, it would be advisable also to get PWD themselves more involved 
and asked them how they experience the use of lifestyle-monitoring systems.

\section{Strengths AND limitations}

One major strength of this study is the number of participants (63 ICs, 13 CMs), which far exceeds that of previous studies. The method we employed provides qualitative information about the concept of lifestyle monitoring, but the results might be biased due to the particular system

\section{Acknowledgements}

Ethical considerations: Ethical challenges are an integral part of this study, and they are mentioned in the various sections of this article.

Contributions: All of the authors approved the final version of this text and met at least one of the following criteria (recommended by the ICMJE): (1) Making substantial contributions to the conception or design of the work, or the acquisition, analysis, or interpretation of data for the work; and (2) Drafting the work or revising it critically for important intellectual content.

\section{Competing interests: None.}

We would like to thank all of the participating organizations for their support and commitment during the investigation, particularly Tinz, de KwadrantGroep, Thuiszorg Het Friese Land and ZuidOostZorg. We are also grateful to ZonMw for their financial contribution within the public-private Breakthrough Project "De zorg ontzorgd met ICT" [Using IT to reduce concerns in care] (2015-2016).

\section{References}

ADI Consortium. (2009). World alzheimer report 2009: The global prevalence of dementia. Retrieved from http://www.alz.co.uk/

Aguglia, E., Onor, M. L., Trevisiol, M., Negro, C., Saina, M., \& Maso, E. (2004). Stress in the caregivers of alzheimer's patients: An experimental investigation in italy. American Journal of Alzheimer's Disease and Other Dementias, 19(4), 248-252. Doi:10.1177/153331750401900403

Atchley, R. C. (1989). A continuity theory of normal aging. The Gerontologist, 29(2), 183-190.

Chi, N. C., \& Demiris, G. (2015). A systematic review of telehealth tools and interventions to support family caregivers. Journal of Telemedicine and Telecare, 21(1), 37-44. Doi:10.1177/1357633X14562734

Chorus, A. M. J., Perenboom, R. J. M., Hoffstetter, H., \& Stadlander, M. C. (2014). Indicatie van de zorgvraag in 2030: Prognoses van functioneren en chronische aandoeningen: Amsterdam. Leiden: TNO.

Eriksson, I., Gustafson, Y., Fagerstrom, L., \& Olofsson, B. (2010). Prevalence and factors associated with urinary tract infections (UTIs) in very old women. Archives of Gerontology and Geriatrics, 50(2), 132135. Doi:10.1016/j.archger.2009.02.013

Gibson, G., Newton, L., Pritchard, G., Finch, T., Brittain, K., \& Robinson, L. (2016). The provision of assistive technology products and services for people with dementia in the United Kingdom. Dementia (London, England), 15(4), 681-701. Doi:10.1177/1471301214532643
(Livind) that was used. Although the original research goals also included the collection of longitudinal quantitative data, the sample size and the data collected were insufficient to justify any valuable conclusions. Another weakness of the study is that only people who agreed to participate were included. This might have resulted in bias, as the participants are likely to have had a positive attitude with regard to the use of a lifestyle-monitoring system.

Hedman, A., Lindqvist, E., \& Nygård, L. (2016). How older adults with mild cognitive impairment relate to technology as part of present and future everyday life: a qualitative study. BMC Geriatrics, 16, 73. Doi:10.1186/s12877-016-0245-y

Laparidou, D., Middlemass, J., Karran, T., \& Siriwardena, N. (2016). Challenges for carers of people with dementia and their support needs from health and social care providers: A qualitative study.

MacNeil Vroomen, J., Van Mierlo, L. D., van de Ven, P. M., Bosmans, J. E., van den Dungen, P., Meiland, F. J., Droes, R. M., Moll van Charante, E. P., van der Horst, H. E., de Rooij, S. E., \& van Hout, H. P. (2012). Comparing dutch case management care models for people with dementia and their caregivers: The design of the COMPAS study. BMC Health Services Research, 12, 132-6963-12-132. Doi:10.1186/1472-6963-12-132

Moïse, P., Schwarzinger, M., \& Um, M. -. (2004). Dementia care in 9 OECD countries; a comparative analysis. OECD health working papers, no. 13. Paris: OECD Publishing. Doi:10.1787/485700737071

Nijhof, N. (2013). eHealth for people with dementia in home-based and residential care. Enschede: Universiteit Twente.

Nijhof, N., van Gemert-Pijnen, L. J., Woolrych, R., \& Sixsmith, A. (2013). An evaluation of preventive sensor technology for dementia care. Journal of Telemedicine and Telecare, 19(2), 95-100. Doi:10.1258/jtt.2012.120605

Novitzky, P., Smeaton, A. F., Chen, C., Irving, K., Jacquemard, T., O'Brolchain, F., O'Mathuna, D., \& Gordijn, B. (2015). A review of contemporary work on the ethics of ambient assisted living technologies for people with dementia. Science and Engineering Ethics, 21(3), 707-765. Doi:10.1007/s11948-014-9552-x

Peek, S. T., Wouters, E. J., van Hoof, J., Luijkx, K. G., Boeije, H. R., \& Vrijhoef, H. J. (2014). Factors influencing acceptance of technology for aging in place: A systematic review. International Journal of Medical Informatics, 83(4), 235-248. Doi:10.1016/j. ijmedinf.2014.01.004

Reeder, B., Meyer, E., Lazar, A., Chaudhuri, S., Thompson, H. J., \& Demiris, G. (2013). Framing the evidence for health smart homes and home-based consumer health technologies as a public health intervention for independent aging: A systematic review. International Journal of Medical Informatics, 82(7), 565-579. Doi:10.1016/j.ijmedinf.2013.03.007

Reisberg, B., Ferris, S. H., de Leon, M. J., \& Crook, T. 
(1982). The global deterioration scale for assessment of primary degenerative dementia. The American Journal of Psychiatry, 139(9), 1136-1139. Doi:10.1176/ajp.139.9.1136

Wahl, H. W., Iwarsson, S., \& Oswald, F. (2012). Aging well and the environment: Toward an integrative model and research agenda for the future. The Gerontologist, 52(3), 306-316. Doi:10.1093/geront/gnr154
Yusif, S., Soar, J., \& Hafeez-Baig, A. (2016). Older people, assistive technologies, and the barriers to adoption: A systematic review. International Journal of Medical Informatics, 94, 112-116. Doi:10.1016/j. ijmedinf.2016.07.004. Retrieved from http://www. domoticawonenzorg.nl/dwz/LeefstijlmonitoringLivind.html (accessed 24-11-2016) 